\title{
Saltation through the blind spot
}

\author{
GREGORY R. LOCKHEAD, ROBERT C. JOHNSON, and FRAN M. GOLD \\ Duke University, Durham, North Carolina 27706
}

\begin{abstract}
At least three localization illusions can be produced by varying the time relations between two stimuli. Two of these, phi motion and Békésy's illusion, have been induced in areas lacking receptors. To learn if this also occurred with the third illusion, sensory saltation, people reported their observations when Geldard's visual rabbit was induced by stimulating points around the optic disk. The rabbit crossed the blind spot as well as other portions of the eyes, and the illusory point was often localized within the region of the blind spot. All three illusions can be localized in an area devoid of receptors.
\end{abstract}

Given two points in space, at least three illusions can be produced by varying the times at which each of these points is presented to an observer: (1) Phi, or beta, motion is induced by successively stimulating one point and then the other. The illusion is the appearance of a point which moves from the first to the second locus. Phi has been studied extensively. (2) Békésy's illusion is induced by varying the phase relations of two simultaneously and repetitively stimulated points. This illusion is the report of stimulation between the two loci. As one spectacular example, Békésy reported that vibratory stimulation of the two thighs produces a sensation that moves continuously from one knee to the other, as if it were phi, and that, following practice, "the observer will become convinced that the vibratory sensation can be localized in the free space between the knees.... This experience is a very peculiar one"' $(1967$, p. 223$)$, but is easily replicated. Unlike phi, the illusory stimulation can appear as a stationary and discrete point between the two stimulated loci. A more usual example of apparently this same illusion is the localization of a sound within the head when earphones are worn. (3) Sensory saltation (or leaping), like phi motion, is induced by the successive stimulation of first one and then a second point. The illusion is the report of discrete points of stimulation between the actual points that were stimulated. Sometimes many intervening points are reported. Burtt (1917) attempted to produce tactile phi using vibratory stimulation. One of his subjects likened the phenomenal effect to "a little boy running along"' (p. 375). When the stimulus times are appropriate, only one intervening point is reported. Geldard (1975) and Geldard and Sherrick (1972) determined the conditions necessary to produce this minimum example of sensory saltation. They called this "the leaping rabbit" to describe the impression that the stimulation hops along in discrete steps. "The taps created between stimulus loci are separate and distinct. One can point to them. The rabbit is a Ding an sich" (Geldard, 1975, p. 33).

The interstimulus times chosen determine which of these illusions is reported, and all three types of illusions can be produced on the skin, in vision, and in audition. They appear to be produceable wherever localization is well developed. This led Burtt (1917) and other gestaltists to consider these illusions as originating centrally. Indeed, these illusions must be partly central in nature because they do not always require receptors at the point at which the illusory stimulation is reported. Teuber and Bender (1950) reported that visual phi motion can be induced through an acquired scotoma that is either cortical or retinal in origin. Békésy (1967) reported that a stimulus is located in space, as between the thighs or between the thumb and forefinger of the open hand, where there clearly are no receptors. Geldard (1975) reported that visual saltation can be produced by delivering the two stimulating lights to different eyes. Hence, there need not be a continuous receptor surface to produce these illusions. This paper extends this observation.

The normal eye has a peripheral receptor area, the optic disk, which does not have a continuous receptor surface. The problem of how it is that one does not notice any discontinuity in this area of the visual field has been addressed repeatedly in the history of vision research. Ferree and Rand (1912) suggested that the blind spot is not a "missing" area in the monocular field because the central representation of the retina has differing spatial values in accordance with the proximity to the blind spot. An object that falls on both sides of the optic disk was proposed to be seen as continuous due to the spatial values centrally associated with the retina at those points. Associative theories, on the other hand, assert that there is a process of "filling-in" of the blind spot and that this process is based on judgment or probability (see Helson, 1929, for a discussion of several varieties of this position). Overall, the problem of vision in the blind spot has been approached largely from a spatial orientation.

Because sensory saltation is discrete temporally as well as spatially, it is informative to know how the 
rabbit, or the running boy, behaves when forced to cross the blind spot. If the illusion is seen within this region, then there would be further evidence that processes more central than the receptor surface are involved in the illusion, and also evidence that temporal as well as spatial factors must be considered in understanding perceptions about the optic disk.

\section{THE EXPERIMENT}

The complete procedure necessary to produce visual saltation can be found in Geldard (1975); a general description is given here. Two pulses of stimulation participate; they must be brief and separated in space and in time. As with all motion illusions, the interstimulus interval and the intensity of the flashes are important. By either decreasing the time between pulses or increasing the intensity of the second pulse, within the range that saltation is reported, the illusory pulse is located a greater proportion of the distance between the first and second location.

For the data reported here, stimuli were presented at a viewing distance of $1 \mathrm{~m}$ in a three-channel tachistoscope. An aperture blocked the left eye; right-eye fixation was on a red light-emitting diode located approximately on the horizontal meridian and about $15 \mathrm{deg}$ nasal to the center of the stimulus field. This fixation point provided projection of the central region of the stimulus field onto the area of each subject's optic disk.

There were five daily sessions of approximately $1 \mathrm{~h}$ each. Day 1 was used to construct the individualized stimulus materials. On Days 2 and 3, the timing of the stimulus sequence was varied to explore the types of perceptions in the task. The time sequence that produced the clearest rabbit for each subject was chosen for the experimental trials on Days 4 and 5 .

\section{Method}

Subjects. The subjects were one undergraduate and two graduate women who had not seen the rabbit previously. None reported any vision problems and none needed correction to perform the task.

Stimuli. The stimuli were constructed by punching $.5-\mathrm{cm}$ holes in opaque $12 \times 18 \mathrm{~cm}$ black cards. The blind spot pattern was custom-constructed for each observer in the following manner. Holes were punched near the center of the card. While the observer fixated the red spot, this pattern was flashed by transilluminating the cards with yellow light; all reported dots were then filled in, new dots were created, and the new pattern was flashed again. By punching new holes and closing others, the pattern was successively shaped until it was as large as possible and the subject reported no dots when fixating the red light. Then a reference dot was punched in each corner of the $12 \times 18 \mathrm{~cm}$ card. The final card, across observers, had from 50 to 60 "invisible" holes near the center of the card arranged in a tall ellipse approximately $4 \mathrm{deg}$ wide and four visible yellow reference dots.

Each subject's blind-spot pattern was somewhat unique. In order that the stimulus lights be positioned reasonably near each blind spot, the locations of the stimuli were determined individually by trial and error. Eight stimulus positions were chosen: four dots nasal and four dots temporal to the blind-spot pattern,
}

such that these eight dots were each just seen when the red light was fixated. All stimulus flashes were of white light.

Stimulus presentations. The stimulus display was controlled by five timers that measured an L1 period (location 1 flashed), blank A (darkness), L1' (location1 again), blank B (darkness), and L2 (location 2 flashed). The red fixation point and the yellow blindspot pattern and reference dots were illuminated throughout the session. All stimulus luminances were approximately $.5 \mathrm{~mL}$ and slightly brighter than the reference dots. The subject started the trial by fixating the red light and pressing the foot pedal. She was instructed to be certain that none of the blind-spot pattern was visible and that the reference points were clearly seen before pressing the foot pedal. A trial consisted of as many presentations of the stimulus sequence as the subject required to "draw a picture of what you just saw." After some practice, this was generally two presentations, but occasionally three or one.

Responses. Following each stimulus trial, the subject reported her observations by drawing them as accurately as possible on a $12 \times 18 \mathrm{~cm}$ sheet of paper that contained marks in the four corners corresponding to the four reference dots in the stimulus display. The task was to draw what had been seen relative to the reference locations. The subjects indicated the order of the perceived lights by labeling each thing drawn with a number, $1,2,3, \ldots, \mathrm{n}$.

Stimulus conditions. The stimulus durations were selected independently for each subject by presenting randomly different combinations of pairs of the stimulus locations under different time relations to detect that set of times at which each observer reported the sought illusion most frequently. For C.S., these times were $\mathrm{Ll}=\mathrm{Ll}^{\prime}=\mathrm{L} 2=$ blank $B=50 \mathrm{msec}$; blank $\mathrm{A}=\mathbf{2 5 0} \mathrm{msec}$. For B.N. and L.G., these times were $\mathrm{L} 1=\mathrm{Ll}^{\prime}=\mathrm{L} 2=50 \mathrm{msec}$; blank $A$ $=100 \mathrm{msec}$; blank $B=25 \mathrm{msec}$. These last times are more nearly like the times Geldard reported as reliably producing this visual illusion. Following these determinations, there were 209 data trials across the three subjects. These comprised six randomly interspersed classes of stimulus categories: (1) cross-temporal (L1 was nasal to the blind spot and L2 was temporal), (2) cross-nasal (L1 was temporal to the blind spot, L2 nasal), (3) nasal (L1 and L2 both nasal to the blind spot), (4) temporal (L1 and L2 temporal to the blind spot), (5) singles (L1 and L2 at the same location), and (6) catch trials in which no dot was flashed.

\section{Results}

When two different loci were flashed, the subjects typically drew one or the other of two types of protocols. One was the discrete rabbit: a dot was reported near the veridical location of L1, another dot was reported near L2, and a third (and often fourth) dot was reported between $\mathrm{L} 1$ and $\mathrm{L} 2$ in both location and time. This agrees with the descriptions classified as the visual rabbit by Geldard (1975). The other type of report was described as a "tail" by C.S. and was reported as a series of flashes along a path between L1 and L2. This streaking effect was independently denoted by C.S. and L.G. as a dashed line on their report sheets. Something is perceived along much of the path between L1 and L2, but the "tails" were neither a single, crisply defined dot nor a continuous movement. C.S. described the tail as a pebble falling down steps; a pilot observer likened the illusory perception to raindrops. This agrees with descriptions of the illusion on the skin offered by Burtt (1917). All observers regularly reported one or the other of these perceptions on essentially every trial that $L 1$ and $L 2$ were different.

On the blank catch trials, no observer ever re- 
ported any dots. On the singles trials, in which L1 $=\mathrm{L1}^{\prime}=\mathrm{L} 2$, three temporally discrete flashes were generally reported; B.N. and L.G. sometimes reported fewer than three flashes. C.S. regularly and correctly reported three clear dots in the same location and quite near the actual stimulus location in this condition, but B.N. and L.G. often reported two or three dots in slightly different locations, as if the eye had moved slightly between flashes. As measured from the protocols, these differences were small, averaging less than $1 \mathrm{deg}$ of visual angle and ranging from $.2 \mathrm{deg}$ to a maximum of $2.6 \mathrm{deg}$ on the trials on which they did occur. These results from the singles control condition, plus the fact that no subject reported difficulty keeping the blind-spot pattern invisible while viewing the stimulus sequence, suggest that eye movements were not a large enough factor to explain the obtained results concerning the illusion within the blind spot.

\section{Individual Protocols}

Because there were individual differences in the quality of the illusion investigated, a subject-by-subject summary of the cross-nasal and cross-temporal trials is presented here. Detailed nasal and temporal trial results are omitted for brevity, since they add nothing new. We detected no structural differences in the protocols between trials where both stimulus lights were on the same side of the blind spot, and thus when the illusory stimulation was seen in an area having receptors, and trials where the lights were on opposite sides of the optic disk and the illusory stimulation was seen in an area having no receptors.

Figure 1 displays a tracing of a representative individual protocol from B.N.; the outline of the blindspot pattern and the actual locations of the stimuli

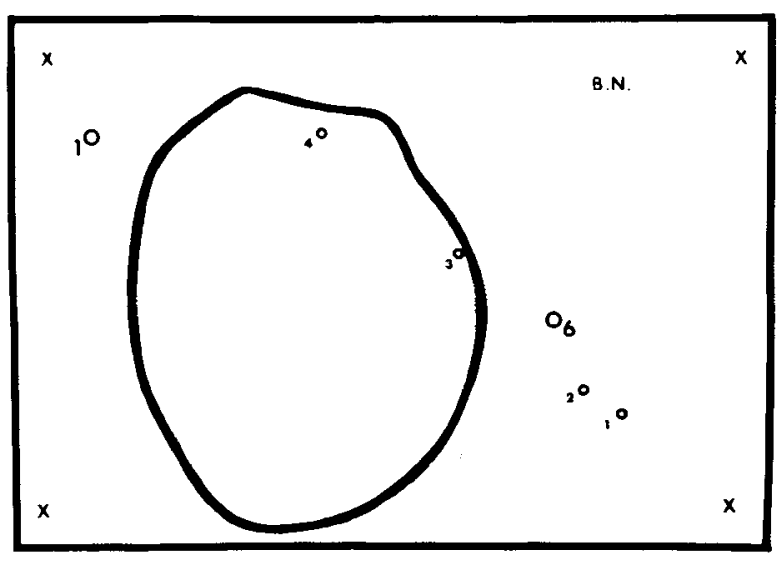

Figure 1. Typical subject protocol (stimulus pair $=6,1$ ). Small circles are locations of perceived dots; ordinal numbers indicate their temporal order. The reference dots appeared as $\times s$ on the protocol sheets. The outline of the blind-spot pattern for B.N. is sketched in, and the large circles are the actual locations of $\mathrm{Ll}$ and $\mathbf{L} 2$.

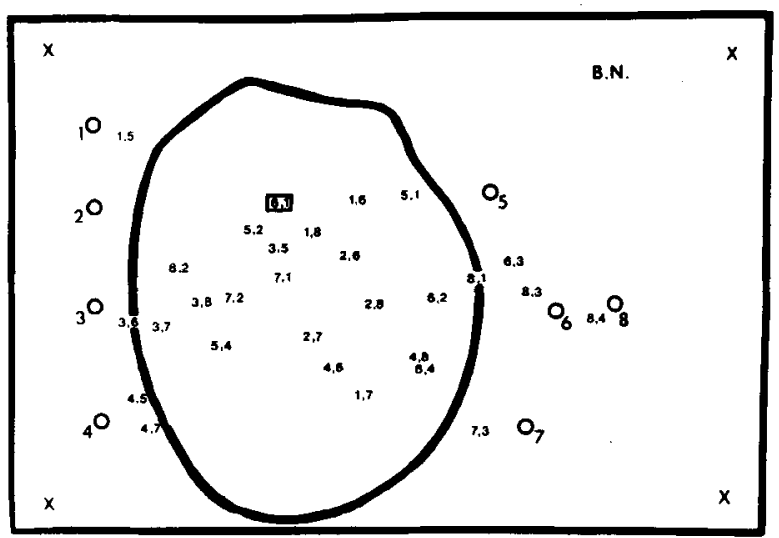

Figure 2. Relative locations of rabbits (next-to-last reported flash) from cross-disk trials for B.N. Numerals indicate stimulus pairs; commas indicate perceived locations of rabbits relative to the size of the stimulus array. The blind-spot region has been sketched in.

on that trial, light 6 followed by light 1 , have been added to the figure. B.N. reported four distinct lights on this trial in the locations and order shown. Response lights 1 and 2 are assumed to be reports of $\mathrm{Ll}$ and $\mathrm{Ll}^{\prime}$, the third dot seen is the rabbit, the fourth light is assumed to be the report of L2. Throughout this report, the illusory light is defined as the dot reported next to last in the protocols; trials when only one or two dots were reported are not represented in any of the figures.

There is one effect in Figure 1 and in many other protocols from all three subjects that was not predicted. On $73 \%$ of all trials in which L1 and L2 differed, L1 was more accurately localized on the response sheet than was L2. Also, there was a tendency for $\mathrm{L} 2$ to be reported as too close to $\mathrm{L} 1$, as if the distance between the points was underestimated and as if $\mathrm{Ll}$ served as the reference location. This is seen in Figure 1, where the last reported dot, presumed here to represent $\mathbf{L} 2$, was relatively close to $\mathrm{L} 1$. Indeed, in this and in many other protocols, L2 was located within the region of the blind spot. This may be a response bias or it may be an important indicator of the relations between receptors about the blind spot and localization in space.

Because observers sometimes reported L2 as being in the blind spot where they could not have seen it, the reported localizations of the illusory lights are presented here relative to the veridical locations of L1 and L2. This conserves the spatial relations in the protocols while showing the nature of the reported perceptions between $\mathrm{L}_{1}$ and $\mathrm{L} 2$. As an example of this measure, the illusory dot in Figure 1 is $56 \%$ of the distance between the reported L1 and L2 (responses 1 and 4). Hence, we report this illusory dot as $56 \%$ of the distance between the veridical L 1 and L2 (light positions 6 and 1); this is the reported illusory dot for B.N. that is outlined in Figure 2. Lo- 
calizations of the illusory lights are reported below in this manner for all observers. The conclusions from this analysis, as they concern the relation between the rabbit and the blind spot, are no different from those when the actual protocol is reported; in either case, most of the illusory spots resulting from the crossdisk trials are within the region of the optic disk.

B.N. For subject B.N., distinct rabbits were reported on $100 \%$ of the cross-temporal trials, $93 \%$ of the cross-nasal trials, $100 \%$ of the nasal trials, and $73 \%$ of the temporal trials. All eight stimuli were regularly detected and reported as dots.

The mean location of the rabbit, defined as the next to the last dot reported on the protocol, can be expressed as a proportion, $\mathbf{R}$ (rabbit), of the distance between the first and last dot positions reported. For cross-temporal trials, $\mathrm{R}=.35(\mathrm{SD}=.22)$; for crossnasal trials, $R=.39(\mathrm{SD}=.26)$; for nasal trials, $\mathrm{R}$ $=.48(\mathrm{SD}=.20)$; and for temporal trials, $\mathrm{R}=.24$ $(\mathrm{SD}=.19)$.

The position of the rabbit relative to $L 1$ and $L 2, R$, for all cross-disk trials of Days 4 and 5 in which the illusion was reported is shown in Figure 2. There is considerable scatter in the locations of the rabbit about the disk, but $69 \%$ of the rabbits were localized within the blind-spot pattern. When the protocol responses of B.N. are directly plotted, without adjusting for the mislocation of L2, at least $52 \%$ of the rabbits are still well within the disk region. The rabbits additionally reported outside the region by this method were mostly reported slightly to the right of the disk; this may be a responding error, since on every one of those trials L2 was reported slightly to the right of its veridical position and, thus, was reported in the blind spot where it could not have been seen. L1 was also reported to the right of its veridical position on those trials. B.N. tended to report the entire pattern as more centered within the reference dots than it actually was. Probably more rabbits were seen within the disk, then, than this response method shows. Regardless of this response bias, the rabbit was a regular and compelling phenomenon for B.N.

L.G. Trials involving stimulus location 4 are not included in the analysis of L.G.'s protocols; this position was seen at best as a faint blur during the experimental sessions and was often not seen at all. It may be that L.G.'s blind-spot pattern was incomplete and that stimulus location $\mathbf{4}$ was actually very near the perimeter of the blind spot, or, as suggested by an anonymous reviewer, there might have been a large blood vessel at this site in the eye. The remaining seven stimulus locations were seen regularly. In crossed trials in which both stimulus locations were reported as either clear dots or as dots with tails, L.G. reported rabbits on $100 \%$ of the presentations. Also, $100 \%$ of the nasal trials and $66 \%$ of the temporal trials produced rabbits. L.G. reported tails on all but one of the cross-trial rabbits. The $R$ values for

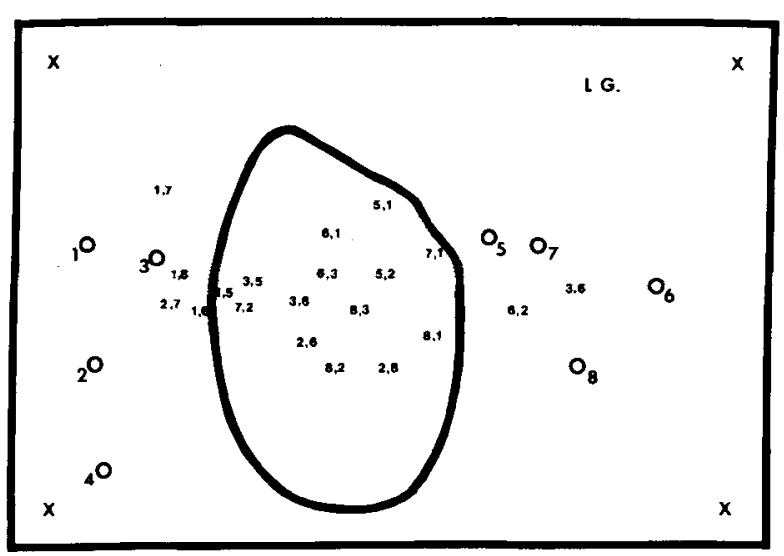

Figure 3. Relative locations of rabbits from cross-disk trials for L.G.

L.G. were as follows: cross-temporal trials, $R=.36$ $(\mathrm{SD}=.21)$; cross-nasal trials, $\mathrm{R}=.41 \quad(\mathrm{SD}=.18)$; nasal trials, $\mathrm{R}=.59(\mathrm{SD}=.14)$; and temporal trials, $\mathrm{R}=.36(\mathrm{SD}=.29)$. Figure 3 shows that $70 \%$ of the cross-disk rabbits were localized within the blindspot pattern as estimated by the relative distance of each illusory dot to L1 and L2; $60 \%$ are within the blind-spot region when the protocols are plotted directly. As with B.N., L.G. found the illusion compelling whenever the two stimulus dots were clearly seen.

C.S. On trials when two different stimulus locations were flashed, C.S. generally had difficulty seeing anything other than the second class of reported illusions, a streaming of white light toward L2. She usually reported two dots near the locus of $\mathrm{Ll}$ ( $\mathrm{L1}$ and $\mathrm{Li}^{\prime}$ ) that were followed in time by a streaming of dots between L1 and L2. This streaming ended near the last reported dot position, assumed here to be the report of L2. The stimulus sequence used for C.S. was longer than the sequences used for the other observers and was more like the time intervals which produce phi motion.

On $96 \%$ of the cross-disk trials, C.S. reported light within the region of the blind spot according to the adjusting measure; this is also the case on $84 \%$ of the trials when no response bias correction is made. The typical protocol was of $\mathrm{L} 1$ and $\mathrm{L} 2$ and a tail between them. Figure 4 shows several representative protocols adjusted to the veridical stimulus locations. The numbers on each protocol report the stimuli for that trial and the lines show what C.S. reported between the reported positions of $\mathrm{L} 1$ and $\mathrm{L} 2$.

\section{CONCLUSIONS}

Our subjects regularly reported the perception of illusory stimulation in the region of the blind spot. The illusion was compelling; indeed, one subject asked how it was that she could see the single white 


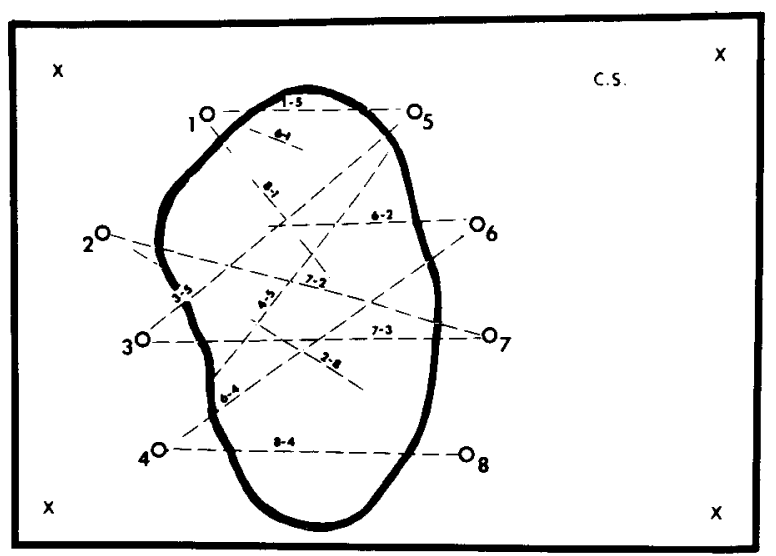

Figure 4. Representative samples of C.S.'s protocols from cross-disk trials scaled to the actual size of the stimulus array. Stimulus pairs are indicated above the reported "tails" (dashed lines).

flashes of light in the same place that a matrix of yellow dots was invisible. Forcing the rabbit to cross the optic disk apparently has no effect on this illusion; receptors are not necessary at the site in which the (illusory) light is seen.

A salient characteristic of sensory saltation is that the illusion is often a discrete point. Since this illusion is seen within the area of the blind spot, theories to explain the "filling in" of spatial or contour information about the optic disk must consider discrete as well as continuous perceptions within that region. One possibility, whatever the basis of the visual rabbit, and probably also of all motion illusions, is that the illusory sequences occur within neural structures that integrate spatial information over time and accomplish this across areas which themselves do not, necessarily, make any neural contribution. Perhaps even the normal mode of perception is to integrate across areas whether or not there are receptors or there is stimulation at every site (see Arend, Buehler, \& Lockhead, 1971).
The illusory "tail"' (see Figure 4) further suggests a continuum between these illusions. When Burtt (1917) induced tactual phi with a similar procedure, his subjects' reports ranged from " $a$ number of intermediate points but also a connection," to "a number of stimuli in a row 'pretty close but possibly felt as separate' from a continuous flow," to where the subject "could not tell the separate points" (p. 375). This tactual illusion sounds much like the visual effects reported here, and these illusions may be closely related. All of these motion illusions depend upon two spatially separated stimulus events. Depending on the temporal characteristics of those stimulations, reports ranging from the continuous movement of a point, to the appearance of many moving points, to several stationary points, to a single stationary point in space may occur.

\section{REFERENCES}

Arend, L. E., Buehler, J. N., \& Lockhead, G. R. Difference information in brightness perception. Perception \& Psychophysics, 1971, 9, 367-370.

BÉKÉSY, G. VON. Sensory inhibition. Princeton: Princeton University Press, 1967.

BURTT, H. E. Tactual illusions of movement. Journal of Experimental Psychology, 1917, 2, 371-385.

Ferree, C. E., \& $R_{A N D}, G$. The spatial values of the visual field immediately surrounding the blind spot and the question of associatively filling in of the blind spot. American Journal of Psychology, 1912, 29, 398-417.

GeLdard, F. A. Sensory saltation: Metastability in the perceptual world. Hillsdale, N.J: Erlbaum, 1975.

Geldard, F. A., \& Sherrick, C. E. The cutaneous "rabbit": A perceptual illusion. Science, 1972, 178, 178-179.

HeLson, $H$. The effects of direct stimulation of the blind spot. American Journal of Psychology, 1929, 41, 345-397.

Teuber, H. L., \& Bender, M. B. Perception of apparent movement across acquired scotomata in the visual field. American Psychologist, 1950, 5, 271. (Abstract)

(Received for publication September 24, 1979; revision accepted March 5, 1980.) 